\title{
Mechanical Oscillation and Cooling Actuated by the Optical Gradient Force
}

\author{
Qiang Lin, Jessie Rosenberg, Xiaoshun Jiang, Kerry J. Vahala, and Oskar Painter* \\ Thomas J. Watson, Sr., Laboratory of Applied Physics, California Institute of Technology, Pasadena, California 91125, USA
} (Received 24 May 2009; published 31 August 2009)

\begin{abstract}
In this work, we combine the large per-photon optical gradient force with the sensitive feedback of a high quality factor whispering-gallery microcavity. The cavity geometry, consisting of a pair of silica disks separated by a nanoscale gap, shows extremely strong dynamical backaction, powerful enough to excite coherent oscillations even under heavily damped conditions (mechanical $Q \approx 4$ ). In vacuum, the threshold for regenerative mechanical oscillation is lowered to an optical input power of only $270 \mathrm{nW}$, or roughly 1000 stored cavity photons, and efficient cooling of the mechanical motion is obtained with a temperature compression factor of nearly $14 \mathrm{~dB}$ with an input optical power of only $11 \mu \mathrm{W}$.
\end{abstract}

The use of laser light to control mechanical motion has been extensively studied for nearly four decades [1], and plays a critical role across many fields from atomic physics [2] to molecular biology [3]. Similarly, in the measurement of weak forces [4], the electromagnetic field in an interferometer or cavity can be coupled to mechanical degrees of freedom to provide exquisite displacement sensitivity. Today, cavity-mechanical systems span a wide range of geometries and scales, from multikilometer long gravitational-wave detectors [5] to coupled nanomechanical-microwave circuits [6]. For the sensitive detection and actuation of mechanical motion, each of these systems depends upon "dynamical backaction" [7] resulting from the dependence of the internal cavity field on mechanical motion and the exertion of radiation pressure by the cavity field back on the mechanical system. Recent work in the optical domain has used the scattering radiation pressure force to both excite and dampen oscillations of a micro-mechanical resonator [8-12], with the intriguing possibility of self-cooling the mechanical system down to its quantum ground-state.

The optical force exerted by a photon inside a resonant cavity is given by $\hbar g_{\mathrm{OM}}$, where $g_{\mathrm{OM}} \equiv d \omega_{c} / d x$ is a coefficient characterizing the dispersive nature of the cavity with respect to the mechanical displacement, $x$. In conventional optomechanical cavity geometries such as the FabryPerot [Fig. 1(a)] or microtoroid whispering-gallery resonator [Fig. 1(b)], the optical force manifests itself as a socalled scattering radiation pressure due to direct momentum transfer from the reflection of photons at the cavity boundary [13]. Alternatively, it has recently been realized [14] that optical intensity gradients found in the near-field of guided wave nanostructures can be harnessed to create large gradient-like optical forces [1]. In contrast to the scattering force, the gradient force depends upon the transverse evanescent-field coupling between adjacent cavity elements, effectively decoupling the strength of the force from the round-trip cavity length and allowing for photon momentum to be transferred over a length scale approaching that of the wavelength of light itself [15]. In this work, we present a nano-optomechanical system (NOMS) which combines the large per-photon optical gradient force with the sensitive feedback of a high quality factor whisperinggallery microcavity. The cavity geometry, shown in Fig. 1(c) and consisting of a pair of silica disks separated by a nanoscale gap, shows extremely strong dynamical backaction, orders of magnitude larger than in other optomechanical systems $[8-12,15-17]$.

One way to quantify the strength of dynamic backaction effects in a cavity optomechanical system is to consider the magnitude of the amplification/damping that an input laser imparts on the mechanical motion. The rate of amplification or damping can be written as [13]

$$
\Gamma_{\mathrm{OM}}=\frac{2 g_{\mathrm{OM}}^{2} P_{i}}{\omega_{c} m_{x} \kappa_{i}^{3} f(\Delta)}
$$

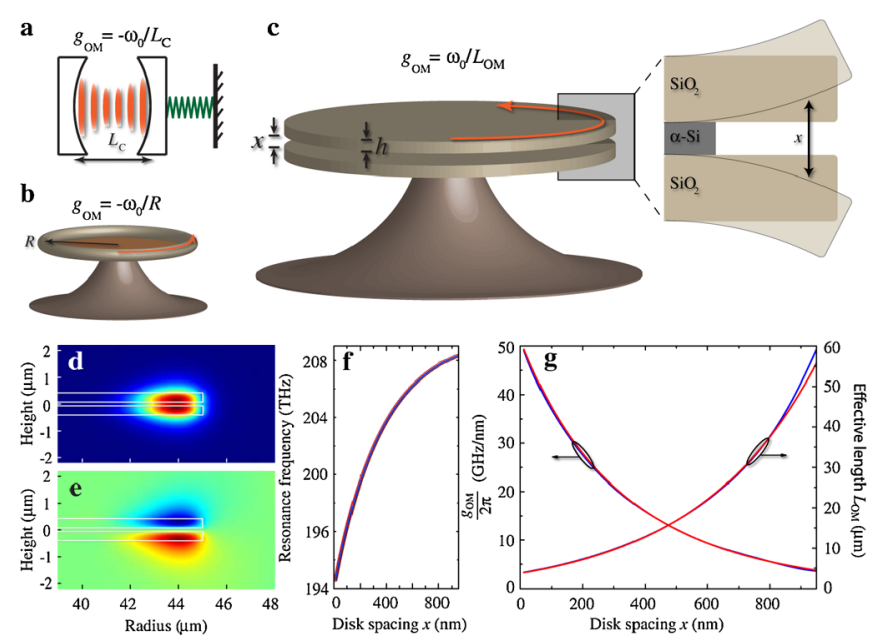

FIG. 1 (color online). Schematic of the corresponding (a) Fabry-Perot and (b) microtoroid optomechanical cavities. (c) Schematic of the double-disk NOMS structure. FEMsimulated optical mode profiles of the radial component of the electric field for the (d) bonded mode at $\lambda=1520 \mathrm{~nm}$ and (e) antibonded mode at $\lambda=1297.3 \mathrm{~nm}$. (f) FEM-simulated tuning curve of the bonded mode. (g) Optomechanical coupling coefficient and effective length (blue curves) for the bonded mode. 
where $P_{i}$ is the optical input power, $\kappa_{i}$ is the intrinsic photon decay rate, and $f(\Delta)$ is a normalized detuning function accounting for the feedback of the optical cavity. In the bad cavity or sideband unresolved regime $(\kappa \gg$ $\left.\Omega_{m}\right), f(\Delta)$ is given by [13]

$$
f(\Delta) \equiv \frac{\left[\Delta^{2}+(\kappa / 2)^{2}\right]^{3}}{\kappa \kappa_{e} \kappa_{i}^{3} \Delta},
$$

where $\kappa_{e}$ is the photon escape rate associated with the external coupling, $\kappa=\kappa_{i}+\kappa_{e}$ is the photon decay rate of the loaded cavity, $\Omega_{m}$ is the mechanical resonance frequency, and $\Delta=\omega_{l}-\omega_{c}$ is the laser frequency detuning from the cavity resonance. Under optimal detuning conditions, Eqs. (1) and (2) yield a maximum amplification/damping rate of $\Gamma_{\mathrm{OM}} \propto g_{\mathrm{OM}}^{2} P_{i} /\left(\omega_{c} m_{x} \kappa_{i}^{3}\right)$. The efficiency of the amplification or cooling process can then be quantified by a backaction parameter, $B \equiv$ $g_{\mathrm{OM}}^{2} /\left(\kappa_{i}^{3} \omega_{c} m_{x}\right)$, which depends upon the motional mass, the per-photon force, and the optical cavity $Q$ factor.

The double-disk cavities presented in this work are formed from a silicon wafer on which a multilayer stack consisting of silicon dioxide disk layers separated by a sacrificial amorphous silicon $(\alpha$-Si) layer have been deposited. A dry release of the silica disk layers is performed using a highly selective plasma etch of the $\alpha$-Si intermediate layer and the underlying $\mathrm{Si}$ substrate. The final doubledisk structure, shown in Fig. 2, consists of 340-nm-thick silica disks separated by a $138 \mathrm{~nm}$ air gap extending approximately $6 \mu \mathrm{m}$ in from the disk perimeter (the undercut region). Two different sized cavities are studied here, one large $(D=90 \mu \mathrm{m}$; Sample I) and one small $(D=54 \mu \mathrm{m}$; Sample II) in diameter.

Finite element method (FEM) simulations of the whispering-gallery optical modes of the double-disk structure shows splitting of the cavity modes into even and odd parity bonded and antibonded modes [Figs. 1(e) and 1(f)]. Because of its substantial intensity within the air gap, the bonded mode tunes rapidly with changing gap size as shown in the inset to Fig. $1(\mathrm{~g})$. The optomechanical coupling coefficient, $g_{\text {OM }}$ can be related to an effective optomechanical coupling length, $L_{\mathrm{OM}}$ through the relation $g_{\mathrm{OM}} \equiv \omega_{c} / L_{\mathrm{OM}} \cdot L_{\mathrm{OM}}$ decreases exponentially with disk spacing [Fig. 1(g)], reaching a minimum value of $3.8 \mu \mathrm{m}$ for a resonance wavelength $\sim 1.5 \mu \mathrm{m}$. This should be compared with conventional optomechanical structures such as those shown in Figs. 1(a) and 1(b), for which $L_{\mathrm{OM}}$ is proportional to the round-trip cavity length and is usually many tens of wavelengths in size. For the air gap of $138 \mathrm{~nm}$ used in this work, the optomechanical coupling is estimated to be $g_{\mathrm{OM}} / 2 \pi=33 \mathrm{GHz} / \mathrm{nm}\left(L_{\mathrm{OM}}=\right.$ $5.8 \mu \mathrm{m}$ ), equivalent to a gradient force of $22 \mathrm{fN} /$ photon.

The double-disk structure also supports a number of different micro-mechanical resonances, ranging from radial breathing modes to whispering-gallery vibrations of the disk perimeter. The most strongly coupled mechanical

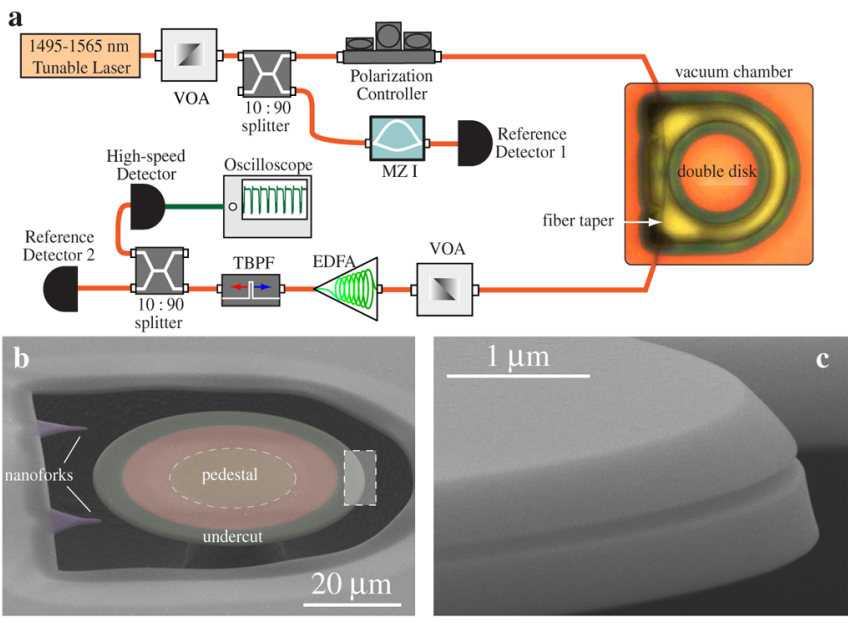

FIG. 2 (color online). (a) Schematic of the experimental setup for optical testing of the double-disk cavity. The cavity input and transmission are both transported through a silica fiber taper, which is supported by two nanoforks for stable operation. A tunable laser source is used to optically probe and actuate the double-disk structure, with input power controlled by a variable optical attenuator (VOA) and wavelength calibrated by a MachZehnder interferometer (MZI). For experiments in vacuum, an erbium-doped fiber amplifier (EDFA) is used to amplify the transmission signal. (b)-(c) Scanning electron microscope images of the 54- $\mu \mathrm{m}$ double-disk NOMS.

resonance is that of the symmetric flapping motion of the disks. The flapping mode exhibits an effective motional mass, $m_{x}$, of only 145 (264) picograms for the 54(90) $\mu \mathrm{m}$ diameter cavity, as predicted by FEM simulations [18].

Optical and mechanical measurements were initially performed in a $1 \mathrm{~atm}$ nitrogen environment. Figure 3(a) shows the wavelength scan of the 90- $\mu \mathrm{m}$ device (Sample I). Several radial-order whispering-gallery modes are evident in the spectrum, all of them with TE-like polarization and bonded mode character. The bonded optical mode at $\lambda=1518.57 \mathrm{~nm}$ is shown in the inset to Fig. 3(a), from which an intrinsic optical $Q$ factor of $1.75 \times 10^{6}$ is inferred [18]. The radio-frequency (RF) power spectrum of the detuned transmitted optical signal for this cavity resonance is shown in the top panel of Fig. 3(b). The main, broad RF resonance peak at $8.3 \mathrm{MHz}$ corresponds to the symmetric differential flapping mechanical mode of the double-disk structure. The 3-dB linewidth of this resonance is approximately $\Gamma_{m}=2.1 \mathrm{MHz}$ (mechanical $Q$ factor, $Q_{M}=3.95$ ), a result of the strong damping caused by the squeeze-film effect of the trapped nitrogen gas between the disks [19]. A model of the transduced Brownian motion of the differential flapping motion is shown as an overlayed solid curve, capturing both the broadband spectral background and second harmonic shoulder present in the measured spectrum (the narrow peak at $13.6 \mathrm{MHz}$ and the broader manifold of resonances near $27.9 \mathrm{MHz}$ match the FEMsimulated first and second order radial breathing mode frequencies, respectively). 

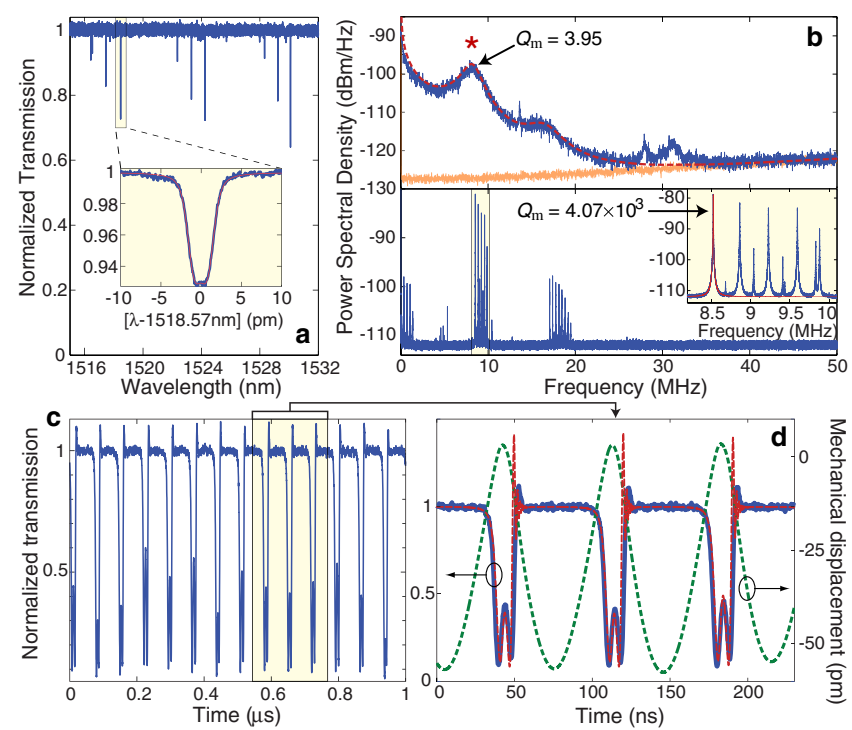

FIG. 3 (color online). (a) Optical transmission spectrum Sample I double-disk cavity. The inset shows the fundamental TE-like bonded mode at $\lambda=1518.57 \mathrm{~nm}$ [18]. (b) Upper panel: optical transmission power spectral density (PSD) of Sample I double-disk in the 1 atm. nitrogen environment for $P_{i}=$ $5.8 \mu \mathrm{W}$. Overlayed dashed curve is the theoretical model. Detector noise background at $-125 \mathrm{dBm} / \mathrm{Hz}$ is shown for reference. Lower panel: transmission PSD of Sample II double-disk cavity in vacuum for $P_{i}=44 \mathrm{nW}$. The inset shows a zoomed-in of the spectrum around the fundamental flapping mode frequency. (c) Recorded transmission waveform of Sample I for $P_{i}=0.76 \mathrm{~mW}$. (d) Comparison of experimental (solid curve) and simulated (dashed curve) waveforms.

Despite the near-unity quality factor of the flapping mode, the powerful dynamic backaction in the doubledisk structure is sufficient to excite regenerative mechanical oscillation. As shown in Fig. 3(c), with an input optical power of $0.76 \mathrm{~mW}$ launched at the blue detuned side of the resonance, the induced parametric mechanical instability causes the cavity transmission to oscillate over the entire coupling depth with a fundamental frequency of 13.97 MHz (this value is about $68 \%$ larger than the intrinsic mechanical frequency due to the optical spring effect [20]). A zoom-in of the recorded time waveform [Fig. 3(d)] agrees well with our numerical simulation which shows that the gradient force actuates an extremely large $(50 \mathrm{pm})$ mechanical displacement amplitude (the overshoot of the transmitted optical power results from the quick release of Doppler shifted photons from the cavity).

The threshold for regenerative oscillation depends upon the optical input power and the laser-cavity detuning, a map of which can be used to quantify the strength of the dynamic backaction. An estimate of the threshold detuning $\left(\Delta_{\text {th }}\right)$, for a given input power, can be determined from the abrupt kink in the cavity transmission that marks the onset of regenerative oscillation [see top panel of Fig. 4(a)]. The detuning dependence of optomechanical amplification is given by the single detuning function, $f\left(\Delta_{\mathrm{th}}\right)$, described above in Eq. (2). The right panel of Fig. 4(b) shows the measured map of $f\left(\Delta_{\mathrm{th}}\right)$ versus optical input power for the $90 \mu \mathrm{m}$ diameter device in the damped nitrogen environment. The data in Fig. 4(b) can be fit with the linear relation $f\left(\Delta_{\text {th }}\right)=2 B P_{i} / \Gamma_{m}$, obtained from Eq. (1) with the oscillation condition $\Gamma_{\mathrm{OM}}=\Gamma_{m}$. The fit yields a dynamic backaction parameter of $B=0.061 \mathrm{MHz} / \mu \mathrm{W}$, corresponding to an optomechanical coupling factor of $g_{\mathrm{OM}} / 2 \pi=$ $33.8 \pm 0.4 \mathrm{GHz} / \mathrm{nm}$, in good agreement with the simulated result of $33 \mathrm{GHz} / \mathrm{nm}$.

In order to eliminate the squeeze-film damping of the nitrogen environment, we performed experiments on a $54-\mu \mathrm{m}$ device (Sample II) in vacuum with a pressure $<5 \times$ $10^{-4}$ Torr. The significantly reduced mechanical linewidth in vacuum shows that the flapping mode consists of a small cluster of modes [Fig. 3(b), bottom panel]. These modes are a mixture of the lower-lying azimuthal modes, coupled together due to deviations in circularity of the undercut region and support pedestal (at higher optical input power the optical spring effect renormalizes the mechanical modes such that the lowest frequency mode at $8.53 \mathrm{MHz}$ transforms into the symmetric flapping mode, while the rest of the modes decouple from the light field). Figure 4(a) shows a transmission spectrum when the laser is scanned across the cavity resonance. Two distinct regimes, corresponding to optically driven regenerative oscillation (I) and optical damping (II), can be clearly seen in the transduced motional amplitude. With an in-vacuum $Q_{M}=4070$ [Fig. 3(b), inset], the fundamental flapping mode has an extremely low threshold input power for regenerative oscillation. The left panel of Fig. 4(b) shows a plot of the invacuum $f\left(\Delta_{\text {th }}\right)$ versus input power, with a measured minimum threshold power for oscillation of $P_{i}=267 \mathrm{nW}$.

The large mechanical amplification of the double-disk NOMS implies a correspondingly efficient cooling of mechanical motion on the red-detuned side of the cavity resonance. As shown in Fig. 4(c) for Sample II in vacuum, the spectral intensity of the fundamental flapping mode decreases dramatically with increased input power, accompanied by a significant broadening of the mechanical linewidth. Even for the strongest damping levels, the inset to Fig. 4(c) shows good signal to noise for the transduced motion due to the high displacement sensitivity of the doubledisk $\left(7 \times 10^{-17} \mathrm{~m} / \mathrm{Hz}^{1 / 2}\right)$. A measure of the optical cooling can be determined from the integrated area under the displacement spectrum [16,18]. Figure 4(d) plots the inferred temperature, $T_{\text {eff }}$, which drops down to $12.5 \mathrm{~K}$ for a maximum input power of $P_{i}=11 \mu \mathrm{W}$. From the thermo-optic tuning of the cavity resonance, the fraction of optical input power that is absorbed in the silica disks is estimated to be $0.55 \%$. In principle, the effective temperature $\left(T_{\text {eff }}\right)$ is related to the optical damping rate $\left(\Gamma_{\mathrm{OM}}\right)$ through the relation $T_{0} / T_{\text {eff }}=1+\Gamma_{\mathrm{OM}} / \Gamma_{m}$, where $T_{0}=300 \mathrm{~K}$ is the bath temperature. In Fig. 4(d), the solid 

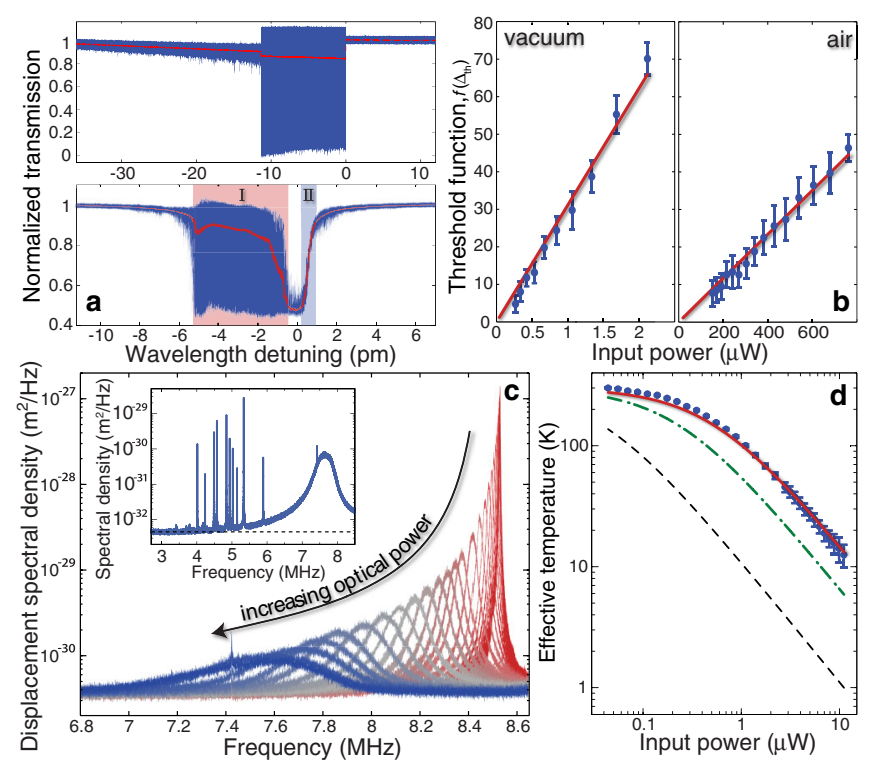

FIG. 4 (color online). (a) Normalized cavity transmission for sample I in a $1 \mathrm{~atm}$. nitrogen environment (top panel) and for Sample II in vacuum (bottom panel) for $P_{i}=0.76 \mathrm{~mW}$ and $11 \mu \mathrm{W}$, respectively. Overlayed solid curve shows the low-passfiltered signal. (b) $f\left(\Delta_{\text {th }}\right)$ as a function of optical input power. Right Panel: Sample I in a $1 \mathrm{~atm}$. nitrogen environment. Left panel: Sample II in vacuum. (c) Spectral intensity of the thermally driven fundamental flapping mode at various input powers, recorded for Sample II in vacuum, with a laser detuning of $\Delta=-1.45(\kappa / 2)$ (inset shows the displacement sensitivity at the highest input power with the second optical attenuator removed), and (d) the corresponding effective temperature. In (d), the solid curve is a fit to the data, the dot-dashed [dashed] curve is a theoretical curve for the experimental [optimal] detuning of $\Delta=-1.45(\kappa / 2)[\Delta=-(\kappa / 2) / \sqrt{5}]$.

curve is a fit of the measured cooling curve using the relation $T_{0} / T_{\text {eff }}=1+\alpha P_{i}$, whereas the dot-dashed curve represents the expected cooling curve for the dynamic backaction parameter $(B=0.032 \mathrm{MHz} / \mu \mathrm{W})$ determined from the threshold plot in the left panel of Fig. 4(b) and the experimental laser-cavity detuning $[\Delta=-1.45(\kappa / 2)]$. For comparison, we have also plotted (dashed curve) the theoretical cooling curve in the case of optimal laser-cavity detuning $[\Delta=-(\kappa / 2) / \sqrt{5})]$. The difference between the two theoretical curves and the measured data, along with the limited range of optical input power studied, can largely be attributed to the limited bandwidth and range of our current cavity locking scheme. As the dashed curve indicates, technical improvements in the cavity locking position and stability should enable temperature compression factors of $20 \mathrm{~dB}$ for less than $3 \mu \mathrm{W}$ of input power.

The large dynamic backaction of the double-disk cavity, primarily a result of the large per-photon force and small motional mass of the structure, opens up several areas of application outside the realm of more conventional ultrahigh- $Q$ cavity geometries. This can be seen by considering not only the efficiency of the cooling or amplification process, but also the maximum rate of effective cooling or amplification, the scale of which is set by the optical cavity decay rate. In the double-disk cavities presented here, the dynamic backaction parameter is $B \approx$ $0.06 \mathrm{MHz} / \mu \mathrm{W}$ for a cavity decay rate of $\kappa / 2 \pi \approx$ $100 \mathrm{MHz}$. The combination allows for high bandwidth operation in heavily damped environments, such as may be found in biological sensing applications [21,22]. In the sideband resolved limit, $B=g_{\mathrm{OM}}^{2} /\left(\Omega_{m}^{3} \omega_{c} m_{x}\right)$, placing even more emphasis on attaining a large $g_{\mathrm{OM}}^{2} / m_{x}$ ratio. Having already achieved optical $Q$ factors in excess of $10^{6}$, and planar silica microdisks having already been demonstrated with $Q>10^{7}$ [23], we expect that further optimization of the double-disk NOMS will be able to extend its operation into the sideband resolved regime.

The authors thank P. Herring and M. Eichenfield for early development of the double-disk structure. This work was supported by a DARPA seedling grant and the NSF (Grant No. 0622246).

*opainter@caltech.edu

[1] A. Ashkin, Phys. Rev. Lett. 24, 156 (1970).

[2] S. Chu et al., Phys. Rev. Lett. 57, 314 (1986).

[3] K. Svoboda et al., Nature (London) 365, 721 (1993).

[4] V. B. Braginskiı̌ and A. B. Manukin, Measurement of Weak Forces in Physics Experiments (University of Chicago Press, Chicago, 1977).

[5] A. Abramovici et al., Science 256, 325 (1992).

[6] C. A. Regal et al., Nature Phys. 4, 555 (2008).

[7] V. B. Braginskiĭ et al., Quantum Measurement (Cambridge University Press, Cambridge, 1992).

[8] T. Carmon et al., Phys. Rev. Lett. 94, 223902 (2005).

[9] S. Gigan et al., Nature (London) 444, 67 (2006).

[10] O. Arcizet et al., Nature (London) 444, 71 (2006).

[11] D. Kleckner et al., Nature (London) 444, 75 (2006).

[12] A. Schliesser et al., Phys. Rev. Lett. 97, 243905 (2006).

[13] T. J. Kippenberg et al., Opt. Express 15, 17172 (2007).

[14] M. L. Povinelli et al., Opt. Express 13, 8286 (2005).

[15] M. Eichenfield et al., Nature (London) 459, 550 (2009).

[16] P. F. Cohadon et al., Phys. Rev. Lett. 83, 3174 (1999).

[17] M. Li et al., Nature (London) 456, 480 (2008).

[18] See EPAPS Document No. E-PRLTAO-103-032936 for additional details of theoretical modeling and data analysis. For more information on EPAPS, see http://www. aip.org/pubservs/epaps.html.

[19] M. Bao et al., Sens. Actuators A, Phys. 136, 3 (2007).

[20] B. S. Sheard et al., Phys. Rev. A 69, 051801(R) (2004).

[21] J. K. H. Hörber and M. J. Miles, Science 302, 1002 (2003).

[22] J. Arlett, Ph.D. thesis, California Institute of Technology, 2006.

[23] T. J. Kippenberg et al., Phys. Rev. A 74, 051802(R) (2006). 